ఠ

\title{
Induction chemotherapy followed by intensity- modulated radiotherapy with reduced gross tumor volume delineation for stage T3-4 nasopharyngeal carcinoma
}

\author{
This article was published in the following Dove Press journal: \\ OncoTargets and Therapy \\ 6 July 2017 \\ Number of times this article has been viewed
}

\section{Fen Xue ${ }^{1,2}$ \\ Chaosu $\mathrm{Hu}^{1,2}$ \\ Xiayun $\mathrm{He}^{1,2}$}

'Department of Radiation Oncology, Fudan University Shanghai Cancer Center, ${ }^{2}$ Department of Oncology, Shanghai Medical College, Fudan University, Shanghai, People's Republic of China
Correspondence: Xiayun He Department of Radiation Oncology, Fudan University Shanghai Cancer Center, Shanghai Medical College, Room 704, Building I, 270 Dong'an Road, Shanghai, People's Republic of China Tel +86 I39 I756 4793 Email hexiayun1962@163.com
Purpose: A common problem in stage T3-4 nasopharyngeal carcinoma (NPC) is the narrow gap between the primary tumor and neurological structures, which makes dose optimization difficult. Considering that significant tumor shrinkage may occur during induction chemotherapy (IC), this study explored the efficacy of intensity-modulated radiotherapy (IMRT) using reduced gross tumor volume (GTV) in the treatment of T3-4 NPC.

Patients and methods: Between January 2009 and April 2014, 103 patients with nonmetastatic stage T3-4 NPC were prospectively recruited. They were assigned to accept IC, followed by reduced-volume IMRT and adjuvant chemotherapy. GTV was based on the post-IC volume of intracavity tumors and lymph nodes, and the pre-IC volume of the remaining involved structures.

Results: For all treated patients, the 3-year local failure-free survival (LFFS) was 91.9\%. After IC, 91 (88.3\%) patients achieved local objective response (OR), and their 3-year LFFS rates were significantly better than in patients who failed to achieve local OR $(94.1 \%$ vs $75.0 \%$, $P=0.023$ ). A multivariate analysis demonstrated the prognostic value of tumor response to IC for LFFS. Dosimetric analysis showed good homogeneity, and the dose constraints were stringent. Asymptomatic temporal lobe necrosis in the ipsilateral side of tumor occurred in one patient.

Conclusion: IMRT using a reduced GTV delineation delivered satisfactory doses to the target volumes and avoided overdosing of critical neurological structures. Results showed satisfactory survival outcomes with few treatment-related toxicities. Tumor response to IC could facilitate selection of patients with stage T3-4 NPC eligible for treatment with this method.

Keywords: nasopharyngeal carcinoma, intensity-modulated radiotherapy, induction chemotherapy, target delineation

\section{Introduction}

Given the anatomical constraints and high degree of radiosensitivity of nasopharyngeal carcinoma (NPC), radiotherapy has been applied as the standard treatment. New radiotherapy techniques, such as intensity-modulated radiotherapy (IMRT), have been widely used in the past decade for highly conformal dose distribution to the primary tumor and reduced dose delivery to organs at risk (OARs), thereby better protecting normal tissues. ${ }^{1,2}$ However, for patients with stage T3-4 NPC, a common problem encountered in planning IMRT is that the space between the primary tumor and neurological structures (eg, the spinal cord, brainstem, optic chiasm, and nerves) is extremely narrow. ${ }^{3,4}$ Thus, it is difficult to optimize the dose in the overlapping region. 
Induction chemotherapy (IC) has been employed in clinical practice for patients with advanced NPC during recent years. With potential advantages in debulking a tumor before irradiation, IC may result in wider spaces between the target volume and normal tissues ${ }^{5}$ and, thereby, improved dose delivery to the primary tumor without exceeding dose limits of OARs can be achieved. The superiority of the docetaxel/cisplatin/5-fluorouracil (TPF) regimen with acceptable toxicities over the cisplatin plus 5-fluorouracil (PF) regimen as IC in patients with head and neck squamous cell carcinoma was demonstrated in the TAX-324 study. ${ }^{6}$ In addition, high response rates $(73.1 \%-91 \%)$ after IC with TPF for NPC have been reported..$^{7-9}$

An innovative study by Lin et $\mathrm{al}^{10}$ revealed that reducedvolume IMRT for NPC ensured the delivery of adequate doses to target volumes and safe doses to OARs, and a high 5-year local control (LC) rate of $95 \%$ was achieved. Therefore, the aim of this study was to explore the efficacy and toxicities of reduced gross tumor volume (GTV) delineation for stage T3-4 NPCs treated with a TPF IC regimen and IMRT.

\section{Patients and methods}

\section{Patient selection and evaluation}

From January 2009 to April 2014, a total of 103 patients with stage T3-4 NPC without distant metastasis were prospectively enrolled in this study at our institution. All patients were newly confirmed pathologically and restaged by MRI as stage T3-4 according to the staging system of the American Joint Committee on Cancer (AJCC 2010). All patients were assigned to receive IC followed by IMRT with adjuvant chemotherapy (AC), according to previous experience in our institute. ${ }^{11}$ IMRT was administered 2 weeks after IC, and AC was administered 1 month after IMRT. IC or AC (TPF regimen) comprised docetaxel $\left(60 \mathrm{mg} / \mathrm{m}^{2} /\right.$ day, day 1), cisplatin ( $25 \mathrm{mg} / \mathrm{m}^{2} /$ day, days 1-3), and 5-fluorouracil $\left(500 \mathrm{mg} / \mathrm{m}^{2} /\right.$ day with a 120 -h infusion) administered once every 3 weeks for two cycles. Patient characteristics are listed in Table 1. The study was approved by the Ethics Committee of Fudan University Shanghai Cancer Center (1410140-8), and written informed consent was obtained from all patients upon recruitment.

The pretreatment evaluation included a complete history and physical examination, fiberoptic nasopharyngoscopy, MRI scans of the head and neck, a CT scan of the chest, a wholebody bone scan, abdominal sonography or CT, as well as hematological and biochemical profiles. Positron emission tomography CT was optional and was performed if necessary. All patients received a dental evaluation before treatment.
Table I Patient characteristics

\begin{tabular}{ll}
\hline Characteristics & $\begin{array}{l}\text { No of patients } \\
(\mathbf{n}=103)\end{array}$ \\
\hline $\begin{array}{l}\text { Age (years) } \\
\text { Median }\end{array}$ & 47 \\
Range & $21-67$ \\
Gender & \\
Male & $72(69.9 \%)$ \\
Female & $31(30.1 \%)$ \\
Histology & \\
WHO I & $0(0)$ \\
WHO II/III & $103(100 \%)$ \\
KPS & \\
70 & $9(8.7 \%)$ \\
80 & $62(60.2 \%)$ \\
90 & $32(31.1 \%)$ \\
T stage & \\
T3 & $60(58.3 \%)$ \\
T4 & $43(41.7 \%)$ \\
N stage & \\
No & $5(4.9 \%)$ \\
NI & $52(50.5 \%)$ \\
N2 & $30(29.1 \%)$ \\
N3 & $16(15.5 \%)$ \\
Overall stage & \\
III & $50(48.5 \%)$ \\
IV & $53(51.5 \%)$ \\
\hline Abreviation
\end{tabular}

Abbreviations: WHO, World Health Organization; KPS, Karnofsky Performance Status.

\section{Image acquisition and registration}

Before radiotherapy, all patients underwent head and neck immobilization with thermoplastic masks and CT simulation with intravenous contrast using a $5-\mathrm{mm}$ slice from the head to a level $2 \mathrm{~cm}$ below the sternoclavicular joint. The contrastenhanced planning CT images obtained were transferred to the Pinnacle ${ }^{3}$ treatment planning system (TPS, Philips Medical Systems, Pinnacle v8.0m, Milpitas, CA, USA) via LAN for target delineation. The pre- and post-IC MRI scans were employed for assisting contour through MRI-CT fusion; contrast-enhanced T1-weighted MRI scans were used. The fusion was based on bony landmarks using automatic registration. Registration accuracy was visually inspected by the operator using the TPS software.

\section{Treatment planning and delivery}

The target volumes were delineated according to the treatment protocol defined as follows: the GTV of the primary tumor (GTV-P) included retropharyngeal lymph nodes, considering the common phenomena of integration, and the rest involved lymph nodes that were defined as GTV-N. For the GTV-P, involved retropharyngeal lymph nodes and intracavity lesions were delineated according to the post-IC 
volume, whereas the remainder of the involved tissues (eg, pterygopalatine fossa) were delineated according to the pre-IC volume of the primary lesion as shown by MRI. Post-IC volumes of involved neck lymph nodes were used for GTV-N delineation (Figure 1). The planning target volume (PTV) of the primary tumor (PTV-G) and the lymph nodes (PTV-N) were created by expanding a 5-mm margin around the GTV-P and the GTV-N. Two clinical target volumes (CTVs) - CTV60 and CTV54 - were then determined. These volumes represented high- and low-risk disease regions, respectively. The CTV60 included the nasopharyngeal cavity, posterior one-third of the nasal cavity and maxillary sinus, parapharyngeal space, pterygopalatine fossa, lateral pterygoid plate, skull base, prevertebral muscles, the whole clivus and sphenoid sinus, retropharyngeal nodal regions, and drainage area of the upper neck (levels II, III, and VA). Adjacent structures were included according to the scope of tumor invasion. The CTV54 included the drainage area of the lower neck (levels IV and VB), which were not outlined for patients with stage N0 or those with retropharyngeal lymph node metastasis only. If the lymph nodes were positive in the level IV or VB, the lower neck was included by the CTV60. PTV60 and PTV54 were created by expanding a 5-mm margin around the CTV60 and CTV54 to compensate for geometric uncertainties and patient movement. A 3-mm gap was created between all the PTVs and the skin surface to minimize dermal toxicity unless the skin was involved or at high risk of tumor involvement. The OARs - including the brainstem, spinal cord, optic nerves, optic chiasma, eyeballs, lenses, temporal lobes, parotid glands, and larynx - were carefully outlined and expanded according to the RTOG 0225 protocol during optimization.

A total dose of 66-70.4 and 66 Gy in 32 fractions was usually prescribed for the PTV-G and PTV-N, whereas 60 and 54 Gy in 32 fractions were prescribed to the PTVs of CTV60 and CTV54. All patients received five daily fractions per week.

The planning goal was to deliver at least $95 \%$ of the prescribed dose to $99 \%$ of the GTV without exceeding the dose tolerance of the critical neurological OARs. It was stipulated that not more than $110 \%$ of the prescribed dose should be outside of the PTV, no more than $5 \%$ of the PTV should receive $\geq 105 \%$ of the prescribed dose, and no more than $3 \%$ of the PTV should receive $\leq 93 \%$ of the prescribed dose. The dose received by each OAR was limited to a tolerance according to the RTOG 0225 protocol, and the dose delivered to $1 \%$ of the volume $\left(\mathrm{D}_{1}\right)$ of neurological tissues (spinal cord, brainstem, temporal lobes, optic nerves, and chiasm) was not to exceed this limitation. By using the TPS, 7-9 fields of a 6-MV photon beam were evenly distributed around each patient's head and neck. The linacs used in this study were Synergy (Elekta, Crawley, UK), with 80 MLCi2 leaves and Trilogy (Varian Medical Systems, Palo Alto, CA, USA) with 120 Millennium MLC leaves.
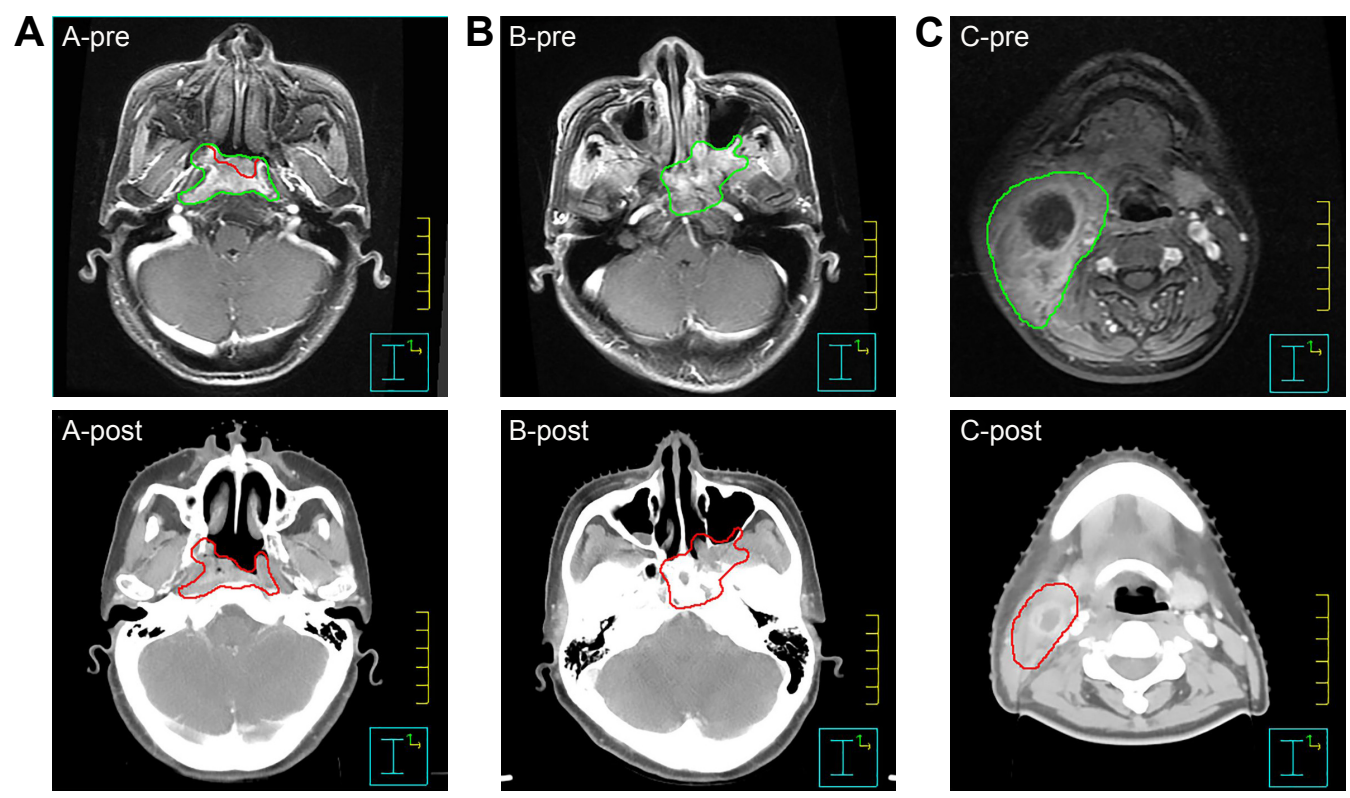

Figure I Examples of target delineation for GTV after induction chemotherapy.

Notes: (A) A-pre presents the tumor (green line) before induction chemotherapy (IC); A-post shows that the regressing part of the intracavitary lesion after IC was not included in GTV (red line). (B) B-pre presents the extension of tumor (green line) before IC; B-post shows that involved tissues (eg, pterygopalatine fossa) were included in GTV (red line) regardless of the regression. (C) C-pre presents the positive lymph node (green line) before IC; C-post shows that the GTV of lymph node (red line) was based on the post-IC volume. 
Inverse-planning software (ADAC Pinnacle 7.4 or 7.6) was used for plan optimization.

\section{Volume and dosimetric analysis}

The dose-volume histograms of the target volumes and OARs were generated to provide the dose and volume data for evaluation. The dosimetric parameters used included the maximum dose $\left(\mathrm{D}_{\max }\right)$, median dose $\left(\mathrm{D}_{\text {med }}\right)$, percent target volume covered by the $95 \%$ prescribed dose line $\left(\mathrm{V}_{95}\right)$, percent target volume covered by the $90 \%$ prescribed dose line $\left(\mathrm{V}_{90}\right)$, dose to $98 \%$ of the target volume $\left(\mathrm{D}_{98}\right)$, dose to $95 \%$ of the target volume $\left(\mathrm{D}_{95}\right)$, dose to $2 \%$ of the target volume $\left(\mathrm{D}_{2}\right)$, and $\mathrm{D}_{1}$. Dose homogeneity was measured by the homogeneity index $(\mathrm{HI})$, which was $\left(\mathrm{D}_{2}-\mathrm{D}_{98}\right) / \mathrm{D}_{\text {med }}$, and a high $\mathrm{HI}$ indicated poor homogeneity.

\section{Follow-up}

Therapeutic effect evaluations were performed via MRI after the completion of IC, IMRT, and AC. All patients were evaluated weekly during treatment, and follow-up visits were required after the completion of treatment at every 3 months during the first 2 years, every 6 months for years 3-5, and annually thereafter. Each follow-up visit included a complete history and physical examination, as well as nasopharyngoscopy and an MRI scan of the nasopharynx. Chest X-ray or CT and abdominal sonography or CT were conducted annually. Additional tests were ordered whenever clinically indicated. The assessment of tumor response was based on MRI data according to the Response Evaluation Criteria for Solid Tumors criteria. ${ }^{12}$ Chemotherapy- and radiotherapy-induced toxicity was assessed and scored according to the National Cancer Institute Common Toxicity Criteria version $3.0^{13}$ and the RTOG radiation morbidity scoring criteria ${ }^{14}$ during treatment and at each follow-up visit. Late toxicity was defined as symptoms or imaging-detected injuries occurring after 3 months or lasting beyond 90 days after treatment completion, and was documented at every follow-up visit.

\section{Statistical analysis}

SPSS 22.0 (SPSS Inc, Chicago, IL, USA) was used for statistical analysis. The actuarial local failure-free survival (LFFS), regional failure-free survival (RFFS), distant failure-free survival (DFFS), and overall survival (OS) were measured from the date of treatment to a documented event or the last follow-up visit. The estimation was performed using the Kaplan-Meier method and log-rank test. Multivariate analyses of significant factors were conducted using the Cox proportional hazards model. A $P$-value $<0.05$ indicated a significant difference.

\section{Results \\ Treatment outcomes}

Most patients (98.1\%) completed the whole IC course; two patients $(1.9 \%)$ discontinued the second cycle of IC due to grade 4 myelosuppression with a poor physical status. All patients completed IMRT at the prescribed dose, except one who was interrupted at $68.2 \mathrm{~Gy} / 31$ fractions due to subarachnoid hemorrhage and another who refused to continue at $63.8 \mathrm{~Gy} / 29$ fractions due to personal reasons. AC was administered to 81 patients $(78.6 \%)$, with one cycle in 14 patients (13.6\%) and two cycles in 67 patients $(65.0 \%)$. Discontinuations of AC were due to grade 4 myelosuppression $(n=7)$, physical intolerance $(n=5)$, and patient refusal due to complete response (CR) of the disease $(n=2)$.

The median follow-up time for the entire group of patients was 50 months (range, $8-85$ months). By the last follow-up visit, a total of 27 patients (26.2\%) experienced treatment failure: 10, 6, and 15 patients developed local, regional, and distant failures, respectively. All locoregional recurrences occurred within the target region. Moreover, 21 (20.4\%) patients had died: five due to local recurrence, seven due to distant metastasis, three due to locoregional recurrence plus distant metastasis, two due to a second primary cancer, and one due to severe late complications; the remaining three patients died of unknown causes. For the entire group, the 3 -year LFFS, RFFS, DFFS, and OS rates were 91.9\%, 97.0\%, $89.9 \%$, and $89.2 \%$, respectively.

\section{Response to induction chemotherapy}

With regard to local response to IC, 91 of the 103 patients (88.3\%) achieved objective response (OR: CR plus partial response [PR]), and 12 patients (11.7\%) maintained stable disease (SD). As regards regional response to IC, OR was observed in $88.8 \%$ (87/98) patients, and SD was observed in $11.2 \%$ (11/98) patients. No progressive disease (PD) was observed after IC. When comparing the local OR group with the local SD group, univariate analysis revealed a significant difference in the 3 -year LFFS rate $(94.1 \%$ vs $75.0 \%$, $P=0.023$; Figure 2), but no differences were observed in the 3 -year RFFS (94.9\% vs 100\%; $P=0.409)$, DFFS ( $89.7 \%$ vs $91.7 \% ; P=0.819)$, or OS rates (91.2\% vs $74.1 \% ; P=0.629$ ). A multivariate analysis with the Cox proportional hazards model was performed to study the significance of various prognostic factors, such as age, gender, $\mathrm{T}$ stage, $\mathrm{N}$ stage, and tumor response to IC. The T stage (hazard ratio [HR] $0.159 ; 95 \%$ confidence interval $[\mathrm{CI}] 0.030-0.842 ; P=0.031$ ) and tumor response to IC (HR $0.2 ; 95 \%$ CI $0.043-0.931$; $P=0.040$ ) were found to be independent prognostic predictors of LFFS. 


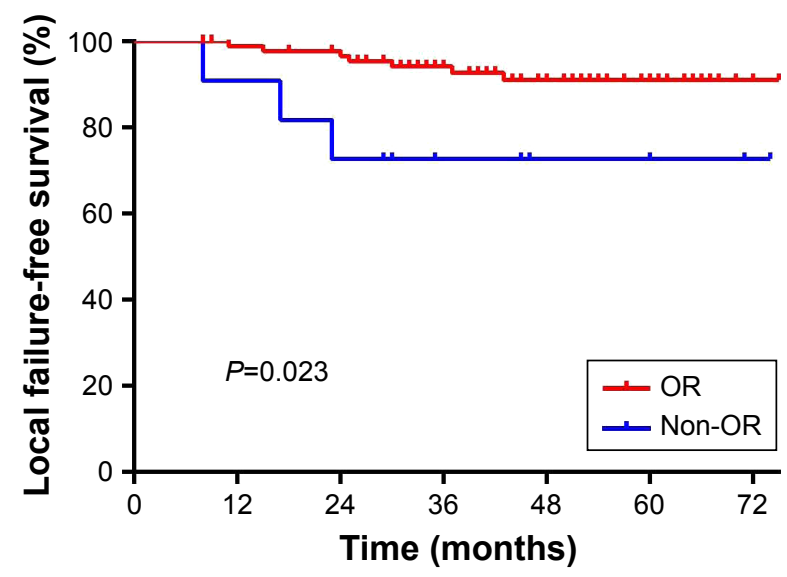

Figure 2 Univariate analysis of patients with local OR and without local objective response (non-OR) after induction chemotherapy on local failure-free survival. Abbreviation: OR, objective response.

\section{Dosimetric analysis}

Dosimetric data are summarized in Tables 2 and 3. Although most patients had GTV-P adequately covered by $95 \%$ of the prescribed dose, approximately $42.7 \%$ of the GTV-P dose distributions did not comply with the planning objectives due to proximity to critical neurological structures. With regard to HI, the results showed that our method of reduced GTV delineation seemed to result in good homogeneity.

All mean $\mathrm{D}_{1}$ values of neurological OARs were well within their dosimetric limitations. The results showed that the maximum $\mathrm{D}_{1}$ values of the brainstem, spinal cord, optic chiasm, optic nerves, and temporal lobes among the patients were $56.2,44.3,59.6,59.9$, and $65.8 \mathrm{~Gy}$, respectively. Only $3.9 \%$ of patients presented with $\mathrm{D}_{1}$ values of the ipsilateral temporal lobes slightly exceeding the tolerance dose of $65 \mathrm{~Gy}$.

\section{Toxicity}

No grade 5 acute toxicity (death) occurred during treatment. The most common grade 3-4 adverse events during

Table 2 Dosimetric parameters of the target volumes in the nasopharynx

\begin{tabular}{lll}
\hline Parameters & GTV-P & PTV-G \\
\hline Volume $\left(\mathrm{cm}^{3}\right)$ & $48.7(5.1-166.9)$ & $83.9(24.3-252.0)$ \\
$\mathrm{V}_{95}(\%)$ & $95.3(62.9-100)$ & $94.9(64.5-100)$ \\
$\mathrm{V}_{90}(\%)$ & $97.4(76.3-100)$ & $97.7(76.8-100)$ \\
$\mathrm{D}_{98}(\mathrm{~Gy})$ & $63.8(51.4-71.3)$ & $64.6(54.0-69.8)$ \\
$\mathrm{D}_{95}(\mathrm{~Gy})$ & $65.4(52.4-71.8)$ & $66.6(56.9-70.8)$ \\
$\mathrm{D}_{\text {med }}(\mathrm{Gy})$ & $72.1(67.4-75.0)$ & $72.3(67.3-74.1)$ \\
$\mathrm{D}_{2}(\mathrm{~Gy})$ & $75.5(69.1-77.7)$ & $75.5(69.1-77.7)$ \\
$\mathrm{HI}$ & $0.16(0.03-0.35)$ & $0.15(0.05-0.32)$ \\
\hline
\end{tabular}

Abbreviations: GTV-P, primary gross tumor volume; PTV-G, planning target volume of the primary tumor; $\mathrm{V}_{95}$, percent target volume covered by the $95 \%$ prescribed dose line; $\mathrm{V}_{90}$, percent target volume covered by the $90 \%$ prescribed dose line; $D_{98}$, dose to $98 \%$ of the target volume; $D_{95}$, dose to $95 \%$ of the target volume; $D_{\text {med' }}$, median dose; $D_{2}$, dose to $2 \%$ of the target volume; $\mathrm{HI}$, homogeneity index.
IC were hematological. Grade 3-4 neutropenia occurred in 44 (42.7\%) patients, followed by leucopenia (22 [21.4\%]). During the IMRT phase, $24.3 \%$ of the patients experienced grade 3 acute mucositis; these cases were uncomplicated, and the patients recovered after anti-inflammation fluid infusion. Hematological adverse events were more frequent in patients who received AC than in those who received IC, with grade 3-4 neutropenia occurring in 38 (46.9\%) patients, and grade 3-4 leucopenia occurring in $30(37.0 \%)$ patients.

Two patients developed cranial nerve palsy that was determined to be induced by local recurrence according to the MRI data. Asymptomatic temporal lobe necrosis in the ipsilateral side of tumor was detected by MRI in one patient at 3 years after treatment. No other neurological dysfunctions, such as brainstem or spinal cord injury, were observed by the last follow-up visit.

\section{Discussion}

Patients with stage T3-4 NPC remain challenging to treat due to the proximity of these tumors to critical tissues or neurological structure involvement in some cases. To improve treatment efficacy while sparing critical OARs, we used IC to shrink the tumor bulk and provide a wider margin for irradiation. A previous report ${ }^{5}$ showed that the GTV declined by an average of $61.4 \%$ after three cycles of IC using cisplatin plus 5-fluorouracil, leading to a minimum dose increase and tumor control probability improvement ( $P=0.002$ ). Besides, regression of bulky lymph nodes after IC could minimize remarkable changes in the contour of the neck during subsequent IMRT. ${ }^{15}$ In this study, IC was administered with a TPF regimen, given its superiority in head and neck squamous cell carcinoma as reported by the TAX-324 study. ${ }^{6}$ The recently published, multicenter, phase $3 \mathrm{RCT}^{16}$ also reported that TPF-based IC significantly improved 3-year failure-free survival and OS with acceptable acute toxicity when administered before radiotherapy in locoregionally advanced NPC.

Lin et $a{ }^{10}$ reported that IMRT with reduced target delineation could minimize the radiation dose to neurological structures such as brainstem, spinal cord, temporal lobes, optic nerves, and chiasm, without sacrificing LC. A previous study in our institution that used IC and replanning IMRT for NPC also showed an encouraging 2-year LFFS rate of 88.2\%. GTV was modified according to the tumor shrinkage shown by repeat MRI before the twenty-third fraction of IMRT. Compared with the IMRT plan without replanning, the doses to the target volumes were quite satisfactory, and the doses to critical OARs were reduced. ${ }^{17}$ Thus, it was supposed that dose optimization in the overlapping region between the tumor 
Table 3 Average dose to the neurological OARs and proportion of patients not fulfilling dosimetric constraints

\begin{tabular}{|c|c|c|c|c|c|c|c|}
\hline$\overline{\text { OARs }}$ & $D_{\max }(G y)$ & $D_{1}(G y)$ & $\begin{array}{l}D_{\max }>45 \mathrm{~Gy}, \\
n(\%)\end{array}$ & $\begin{array}{l}D_{1}>54 \mathrm{~Gy} \\
\mathrm{n}(\%)\end{array}$ & $\begin{array}{l}D_{1}>57 \text { Gy, } \\
n(\%)\end{array}$ & $\begin{array}{l}D_{1}>60 \mathrm{~Gy} \\
\mathrm{n}(\%)\end{array}$ & $\begin{array}{l}D_{1}>65 \mathrm{~Gy}, \\
\mathrm{n}(\%)\end{array}$ \\
\hline Brainstem & $55.7 \pm 1.5$ & $52.5 \pm 2.1$ & - & $23(22.3)$ & $0(0)$ & $0(0)$ & - \\
\hline Spinal cord & $43.7 \pm 1.1$ & $40.5 \pm 3.2$ & $4(3.9)$ & - & & - & - \\
\hline Optic chiasm & $54.0 \pm 6.9$ & $53.8 \pm 6.8$ & - & $69(67.0)$ & $22(21.4)$ & $0(0)$ & - \\
\hline \multicolumn{8}{|l|}{ Optic nerve } \\
\hline (I) & $51.9 \pm 8.3$ & $51.8 \pm 7.8$ & - & $51(49.5)$ & $12(11.7)$ & $0(0)$ & - \\
\hline (C) & $51.4 \pm 8.2$ & $50.9 \pm 8.2$ & - & $49(47.6)$ & $7(6.8)$ & $0(0)$ & - \\
\hline \multicolumn{8}{|l|}{ Temporal lobe } \\
\hline (I) & $64.9 \pm 2.6$ & $63.3 \pm 1.3$ & - & - & - & $99(96.1)$ & $4(3.9)$ \\
\hline (C) & $64.7 \pm 1.3$ & $62.3 \pm 1.8$ & - & - & - & $95(92.2)$ & $0(0)$ \\
\hline
\end{tabular}

Note: All data are presented as the mean \pm SD.

Abbreviations: OARs, organs at risk; $D_{\max }$, maximum point dose; $D_{1}$, maximum dose to $1 \%$ volume; I, ipsilateral side; $C$, contralateral side.

and neurological structures can be safely achieved using reduced GTV delineation after IC. In addition, it was supposed that most local recurrence occurred in the tumor areas that exhibited late responses, and that isolated recurrences in marginal areas were minimal even with suboptimal dose coverage. Wu et a ${ }^{18}$ reported 29 local recurrences in NPC patients treated with radiotherapy, and all of the recurrences were located inside the boost areas. In this study, the 10 local recurrences were all within the PTV-G, further confirmed the safety of reduced GTV delineation. The measured HI values of 0.16 for GTV-P and 0.15 for PTV-G in our study were not inferior to the HI values of $0.09,0.19$, and 0.30 for each PTV reported by Mao et al, ${ }^{19}$ indicating the good homogeneity of this method. With a median follow-up time of 50 months, a satisfactory 3-year LFFS rate of $91.9 \%$ was achieved, which was similar to historical outcome rates ranging between $82.1 \%$ and $91.1 \% .{ }^{20-22}$ Therefore, this method of reduced target delineation after IC was feasible to some extent, but further study is required to confirm these findings.

It is worth mentioning that a high local OR rate of $88.3 \%$ after IC was observed in our study. With a median follow-up period of 50 months, the 3-year LFFS rate was significantly higher for patients who achieved local OR with IC than those who remained classified as having local SD $(94.1 \%$ vs $75.0 \%, P=0.023)$. The prognostic value of tumor response to IC for LFFS was demonstrated in the multivariate analysis. A secondary analysis of a randomized phase 3 clinical trial ${ }^{8}$ also revealed that the 3-year failure-free survival rates in the CR $(88.5 \%$ vs $61.9 \% ; P=0.017)$ and PR $(81.2 \%$ vs $61.9 \%$; $P=0.01)$ groups were obviously higher than that of the SD group, and concluded that the tumor response to IC with a TPF regimen may be helpful in developing individualized treatment strategies for patients with locoregionally advanced NPC. In this context, we recommend our method of reduced GTV delineation for patients with local OR after IC, considering the high LFFS rate of $94.1 \%$. However, the application of this method in those who fail to achieve local OR remains controversial.

In the safety analysis, acute toxicity and late neurological toxicity were the major concerns. Grade 1 or 2 acute toxicity was most frequently observed during treatment. Only $24.3 \%$ of the patients had grade 3 acute mucositis during IMRT, compared with $43 \%-47.8 \%$ in other studies. ${ }^{23,24}$ Although grade 3-4 leucopenia or neutropenia during chemotherapy was common in this study, patient compliance was satisfactory at $78.6 \%$ for AC. Moreover, only $3.9 \%$ of patients exceeded the tolerance dose of 65 Gy for the $\mathrm{D}_{1}$ of the ipsilateral temporal lobes, showing a dramatic decrease as compared with the $40 \%$ reported by Mao et al. ${ }^{19}$ Thus, the incidence of temporal lobe necrosis was low in the present study. Other IMRT series studies ${ }^{25-27}$ have reported incidence rates ranging from $3 \%$ to $16 \%$, whereas only one patient (1\%) developed temporal lobe necrosis in this study. This patient had no related symptoms during the follow-up period, and the lesion was confirmed by MRI 3 years after IMRT. However, there are several limitations. First, this is a single-arm prospective study with relatively small sample size and no well-matched control group. Although diagnosis of temporal lobes necrosis or other late toxicities were based on early signs, symptoms, and image examination, omission diagnosis of some subtle injuries was inevitable. Besides, as these results are based on data and experience from a single institution, further validation is required.

Considering tumor bulk shrinkage occurred during IC, a new method for reduced GTV delineation to gain dose optimization and OAR protection was explored in our study for application in stage T3-4 NPC. The results showed that survival outcomes were satisfactory, with little treatmentrelated acute or late toxicity. In addition, patients who achieved OR after IC seemed to benefit from this method, while the application in those who failed to achieve OR requires further investigation. 


\section{Acknowledgments}

This work was supported by the Science and Technology Commission of Shanghai Municipality (Grant no 14411962400).

\section{Author contributions}

FX planned the study and wrote the article and analyzed image data. $\mathrm{CH}$ and $\mathrm{XH}$ collected data. FX undertook statistical analysis. All authors contributed toward data analysis, drafted and critically revised the article, gave final approval of the version to be published, and agree to be accountable for all aspects of the work.

\section{Disclosure}

The authors report no conflicts of interest in this work.

\section{References}

1. Peng G, Wang T, Yang KY, et al. A prospective, randomized study comparing outcomes and toxicities of intensity-modulated radiotherapy vs. conventional two-dimensional radiotherapy for the treatment of nasopharyngeal carcinoma. Radiother Oncol. 2012;104(3):286-293.

2. Lee AW, Ng WT, Chan LL, et al. Evolution of treatment for nasopharyngeal cancer-success and setback in the intensity-modulated radiotherapy era. Radiother Oncol. 2014;110(3):377-384.

3. Ng WT, Lee MC, Chang AT, et al. The impact of dosimetric inadequacy on treatment outcome of nasopharyngeal carcinoma with IMRT. Oral Oncol. 2014;50(5):506-512.

4. Lee AW, Ma BB, Ng WT, Chan AT. Management of nasopharyngeal carcinoma: current practice and future perspective. J Clin Oncol. 2015; 33(29):3356-3364.

5. Lee AW, Lau KY, Hung WM, et al. Potential improvement of tumor control probability by induction chemotherapy for advanced nasopharyngeal carcinoma. Radiother Oncol. 2008;87(2):204-210.

6. Lorch JH, Goloubeva O, Haddad RI, et al; TAX 324 Study Group. Induction chemotherapy with cisplatin and fluorouracil alone or in combination with docetaxel in locally advanced squamous-cell cancer of the head and neck: long-term results of the TAX 324 randomised phase 3 trial. Lancet Oncol. 2011;12(2):153-159.

7. Ou D, Blanchard P, El Khoury C, et al. Induction chemotherapy with docetaxel, cisplatin and fluorouracil followed by concurrent chemoradiotherapy or chemoradiotherapy alone in locally advanced nonendemic nasopharyngeal carcinoma. Oral Oncol. 2016;62:114-121.

8. Peng H, Chen L, Li WF, et al. Tumor response to neoadjuvant chemotherapy predicts long-term survival outcomes in patients with locoregionally advanced nasopharyngeal carcinoma: a secondary analysis of a randomized phase 3 clinical trial. Cancer. 2017;123(9):1643-1652.

9. Wang HM, Lin CY, Hsieh CH, et al. Induction chemotherapy with dosemodified docetaxel, cisplatin, and 5-fluorouracil in Asian patients with borderline resectable or unresectable head and neck cancer. J Formos Med Assoc. 2017;116(3):185-192.

10. Lin S, Pan J, Han L, et al. Update report of nasopharyngeal carcinoma treated with reduced-volume intensity-modulated radiation therapy and hypothesis of the optimal margin. Radiother Oncol. 2014;110(3): 385-389.

11. Du C, Ying H, Zhou J, Hu C, Zhang Y. Experience with combination of docetaxel, cisplatin plus 5-fluorouracil chemotherapy, and intensitymodulated radiotherapy for locoregionally advanced nasopharyngeal carcinoma. Int J Clin Oncol. 2013;18(3):464-471.
12. Eisenhauer EA, Therasse P, Bogaerts J, et al. New response evaluation criteria in solid tumours: revised RECIST guideline (version 1.1). Eur J Cancer. 2009;45(2):228-247.

13. Trotti A, Colevas AD, Setser A, et al. CTCAE v3.0: development of a comprehensive grading system for the adverse effects of cancer treatment. Semin Radiat Oncol. 2003;13(3):176-181.

14. Cox JD, Stetz J, Pajak TF. Toxicity criteria of the Radiation Therapy Oncology Group (RTOG) and the European Organization for Research and Treatment of Cancer (EORTC). Int J Radiat Oncol Biol Phys. 1995;31(5):1341-1346.

15. Lee AWM, Ng WT, Chan OSH, Sze HCK. If concurrent-adjuvant chemoradiotherapy is beneficial for locoregionally advanced nasopharyngeal carcinoma, would changing the sequence to induction-concurrent achieve better outcome? J Radiat Oncol. 2012; 1(2):107-115.

16. Sun Y, Li WF, Chen NY, et al. Induction chemotherapy plus concurrent chemoradiotherapy versus concurrent chemoradiotherapy alone in locoregionally advanced nasopharyngeal carcinoma: a phase 3, multicentre, randomised controlled trial. Lancet Oncol. 2016;17(11): $1509-1520$.

17. Niu X, Chang X, Gao Y, Hu C, Kong L. Using neoadjuvant chemotherapy and replanning intensity-modulated radiotherapy for nasopharyngeal carcinoma with intracranial invasion to protect critical normal tissue. Radiat Oncol. 2013;8:226.

18. Wu F, Wang R, Lu H, et al. Concurrent chemoradiotherapy in locoregionally advanced nasopharyngeal carcinoma: treatment outcomes of a prospective, multicentric clinical study. Radiother Oncol. 2014; 112(1):106-111.

19. Mao YP, Yin WJ, Guo R, et al. Dosimetric benefit to organs at risk following margin reductions in nasopharyngeal carcinoma treated with intensity-modulated radiation therapy. Chin J Cancer. 2015; 34(5):189-197.

20. Cao CN, Luo JW, Gao L, et al. Update report of T4 classification nasopharyngeal carcinoma after intensity-modulated radiotherapy: an analysis of survival and treatment toxicities. Oral Oncol. 2015; 51(2):190-194.

21. Sun X, Su S, Chen C, et al. Long-term outcomes of intensity-modulated radiotherapy for 868 patients with nasopharyngeal carcinoma: an analysis of survival and treatment toxicities. Radiother Oncol. 2014; 110(3):398-403.

22. He Y, Wang Y, Shen L, et al. Prognostic value of the distance between the primary tumor and brainstem in the patients with locally advanced nasopharyngeal carcinoma. BMC Cancer. 2016;16:114.

23. Xu T, Liu Y, Dou S, Li F, Guan X, Zhu G. Weekly cetuximab concurrent with IMRT aggravated radiation-induced oral mucositis in locally advanced nasopharyngeal carcinoma: results of a randomized phase II study. Oral Oncol. 2015;51(9):875-879.

24. Bossi P, Orlandi E, Bergamini C, et al. Docetaxel, cisplatin and 5-fluorouracil-based induction chemotherapy followed by intensitymodulated radiotherapy concurrent with cisplatin in locally advanced EBV-related nasopharyngeal cancer. Ann Oncol. 2011;22(11): 2495-2500.

25. Zhou X, Ou X, Xu T, et al. Effect of dosimetric factors on occurrence and volume of temporal lobe necrosis following intensity modulated radiation therapy for nasopharyngeal carcinoma: a case-control study. Int J Radiat Oncol Biol Phys. 2014;90(2):261-269.

26. Bakst RL, Lee N, Pfister DG, et al. Hypofractionated dose-painting intensity modulated radiation therapy with chemotherapy for nasopharyngeal carcinoma: a prospective trial. Int J Radiat Oncol Biol Phys. 2011;80(1):148-153.

27. Wang XS, Ying HM, He XY, Zhou ZR, Wu YR, Hu CS. Treatment of cerebral radiation necrosis with nerve growth factor: a prospective, randomized, controlled phase II study. Radiother Oncol. 2016;120(1): 69-75. 


\section{Publish your work in this journal}

OncoTargets and Therapy is an international, peer-reviewed, open access journal focusing on the pathological basis of all cancers, potential targets for therapy and treatment protocols employed to improve the management of cancer patients. The journal also focuses on the impact of management programs and new therapeutic agents and protocols on

patient perspectives such as quality of life, adherence and satisfaction. The manuscript management system is completely online and includes a very quick and fair peer-review system, which is all easy to use. Visit http://www.dovepress.com/testimonials.php to read real quotes from published authors.

Submit your manuscript here: http://www.dovepress.com/oncotargets-and-therapy-journal 